\section{Anomalous small-angle $X$-ray scattering in materials science}

\author{
Günter Goerigk, ${ }^{\text {ab }}$ Heinz- Günter Haubold, Olivier \\ Lyon $^{\mathrm{c}}$ and Jean-Paul Simon ${ }^{\mathrm{de}^{*}}$
}

Institut für Festkörperforschung, Forschungzentrum Jülich; D-52425 Jülich, Germany, ${ }^{b}$ DESY-HASYLAB, D-22603 Hamburg, Germany, 'LURE (CNRS-CEA), BP34, 91988 Orsay Cedex, France, 'Laboratoire de thermodynamique et Physico Chimie Métallurgiques (CNRS-INPG-UJF), BP75, 38402 Saint Martin d'Hères, France, and ${ }^{\circ}$ ESRF (CRG-BM2), BP220, 38043 Grenoble Cedex, France. E-mail: jpsimon@ltpcm.inpg.fr

After the exploratory studies of the 1980s, anomalous small-angle $\mathrm{X}$-ray scattering (ASAXS) is now a mature technique to disentangle complex subjects in materials science: this is illustrated through selected examples.

Keywords: anomalous dispersion, SAXS, materials science

\section{Introduction}

X-ray scattering experiments based on the anomalous variation of a scattering factor near an absorption edge (with tuneable synchrotron radiation sources) are element-selective. Nowadays, this characterization technique is used in physics, chemistry, soft / hard condensed matter or biology, to explore different length scales: the atomic scale in diffraction and in wide angle scattering or the study of nanoscopic / mesoscopic objects in small angle X-ray scattering. Anomalous small angle X-ray scattering (ASAXS) was initiated in biology by Stuhrmann, 1985.

This paper focuses on applications of ASAXS in materials science. At the "Resonant Anomalous X-Ray Scattering" workshop, the possibilities of this element-specific technique were described (Haubold et al., 1994; Simon \& Lyon, 1994). Different ways of using ASAXS were illustrated and perspectives for their development were reviewed. Although analysis is now standard, these synchrotron experiments require fairly strict, time-consuming procedures: they are undertaken when normal SAXS is unable to provide the essential information. In Europe, three beamlines have been optimized for this task: JUSIFA at Hamburg, D2AM at Grenoble and D22 at Orsay (the last mentioned will be upgraded when reinstalled on "Soleil" within $\sim 3$ years). This paper is coauthored by the heads of the three beamlines.

The aim of the present paper is to give instructive examples. The novel opportunities are classified as follows:

(i) Contrast enhancement, combined with the separation of different kinds of objects.

(ii) Composition of phases or determination of specific volume in two-phase materials.

(iii) Coupling ASAXS with anomalous wide angle scattering (AWAXS) or anomalous powder diffraction (AXRD).

\section{ASAXS experiments}

\subsection{Anomalous atomic scattering factors}

Anomalous scattering is a labelling technique since, owing to resonance between absorption and elastic scattering in the vicinity of an absorption edge, rapid variation occurs in the atomic scattering factor of the given element $\left(f(E)=f_{0}+f^{\prime}(E)+\mathrm{i} f^{\prime \prime}(E)\right.$, with $\left.f_{0} \sim Z\right)$. The values of $f^{\prime}$ and $f^{\prime \prime}$ tabulated by Cromer \& Liberman (1970) can be used if the relative energy shift from the edge, $\Delta E / E$, is much larger than the bandwidths of the monochromator and of the edge. In practice, the optimum energy range turns out to be $5 \times 10^{-2}<\Delta E / E$ $<10^{-3}$. For $\Delta E / E \sim<10^{-3}$, the tabulated values $f^{\prime}$ and $f^{\prime \prime}$ should be corrected. This can be achieved by absorption measurements and from the Kramers-Kronig relation, using the DIFFKK program (Cross et al., 1998) or else by ASAXS measurements of the relative intensity variations in a precipitated binary alloy.

\subsection{ASAXS beamlines and experiments}

ASAXS needs a SAXS camera together with synchrotron optics, having easily tuneable monochromatic photon energy without degrading the beam alignment. In this respect, it is far easier to align optics with fixed exit double monochromators (D22 at LURE (Dubuisson et al., 1986, http://www.lure.u-psud.fr) or JUSIFA at DESY (Haubold et al., 1989, http://www.fz.juelich.de/iff/personen /H.-G. Haubold) than optics with vertical \& horizontal focusing (D2AM at ESRF, Ferrer et al., 1998; Simon et al., 1997, http://www.esrf.fr/exp_facilities/BM2/BM2.html).

Most monochromators are made of $\mathrm{Si}_{111}$ or $\mathrm{Si}_{311}$, in order to remove second order harmonic. Nowadays, all beamlines use twodimensional detectors. Anomalous variations being small, stability and linearity are required for all the components in the counting assembly (flux monitors, detector, ...). In addition to the standard SAXS corrections, the efficiency of the counting assembly with respect to photon energy should be evaluated precisely.

\section{Classification of ASAXS results}

\subsection{Contrast enhancement, separation of different kinds of objects}

Differential ASAXS identifies objects that contain the anomalous element as well as other SAXS scatterers: any signal that is independent of the energy can be considered a background. In the early stages of precipitation of Co particles in a $\mathrm{Cu}$ matrix, the contrast of interest, $\left(f_{\mathrm{Cu}}-f_{\mathrm{Co}}\right)^{2}$, is increased (Goerigk et al., 1997).

At the JUSIFA beamline, ASAXS experiments have become increasingly important for catalyst-related research (Bönnemann et al., 2002; Bota et al., 2002; Polizzi et al., 2001; Rasmussen et al., 2000; Benedetti et al., 1997, 1999; Haubold et al., 1995, 1996, 1999; Vad et al., 2002). In particular, in the analysis of size distributions of nanometric catalyst particles in porous support materials, the use of anomalous scattering can be retrieved from that of the background from pores by means of anomalous scattering.

Fig. 1 shows an example of supported Pt catalyst particles in porous carbon electrodes as in electrical fuel cells. The scattering intensity is plotted as function of the scattering vector $q(q=4 \pi \sin \theta / \lambda$ where $\theta$ is half the scattering angle and $\lambda$ the wavelength) (Haubold et al., 1999). Anomalous scattering at two X-ray energies near the $\mathrm{Pt}_{3_{3}}$ - absorption edge was used to vary the scattering amplitudes of the Pt catalyst atoms, while the porosity scattering of the carbon 
support remains unaltered at these energies far from the carbon absorption edge. The measured difference in the scattering intensities $I_{1}$ and $I_{2}$ then results from the catalyst particles alone and can be separated to determine a bimodal log-normal size distribution $P(R)$ for these Pt particles: only $88 \%$ of the platinum catalyst was dispersed in the form of small particles having a mean radii of 0.8 $\mathrm{nm}$. Here, as much as $60 \%$ of the platinum resides on the particle surface. It is thus is in contact with the reactants and is catalytically active. For the low platinum loading of only $1.5 \mathrm{mg} / \mathrm{cm}^{2}$ in this electrode, a huge catalytic active platinum area of about $0.2 \mathrm{~m}^{2}$ per $\mathrm{cm}^{2}$ sample surface is found.
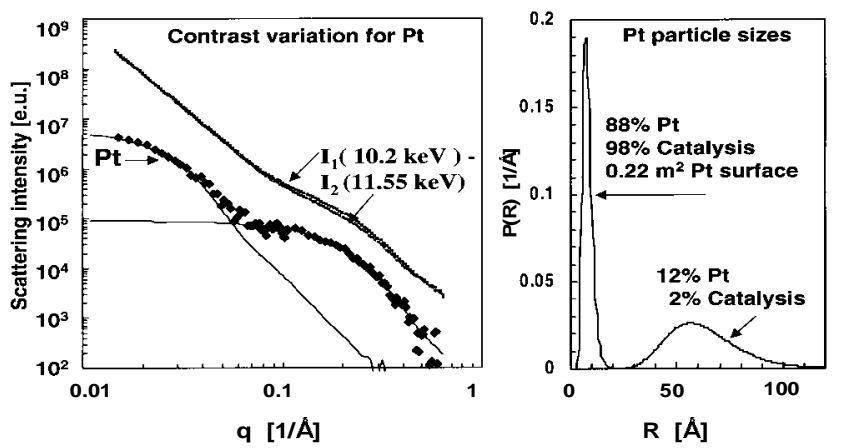

Figure 1

Scattering intensities $I_{1}, I_{2}$ at two X-ray energies with different scattering contrast of Pt particles and intensity difference $I_{1}-I_{2}$ with fits for a bimodal log-normal size distribution $P(R)$ for these particles (Haubold et al., 1999).
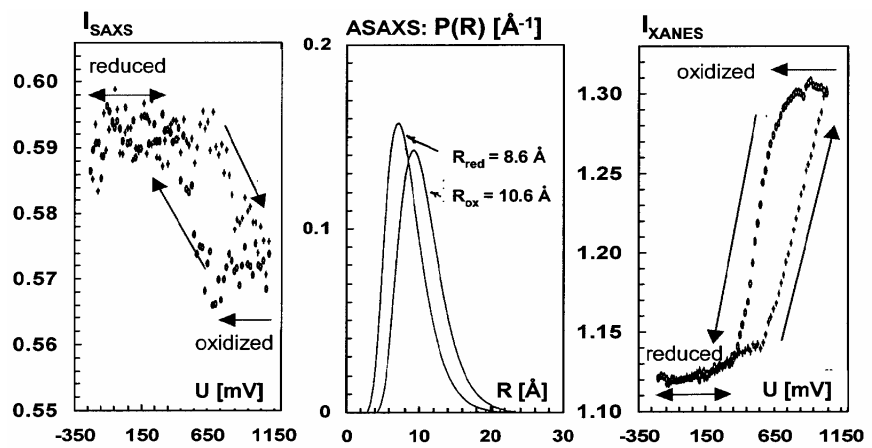

Figure 2

Integrated SAXS intensity, size distributions of Pt particles in the reduced and oxidised state at electro-chemical potentials $250 \mathrm{mV}$ and $1100 \mathrm{mV}$ and "white line" XANES X-ray absorption as measured in a real time potential sweep experiment in an electro-chemical cell (Haubold et al., 1999).

ASAXS had also proved to be an excellent tool for probing the state of catalytically active surface atoms during the course of a catalysed reaction: in the energy range of the absorption edges of noble metal catalysts, X-rays penetrate easily, e.g. even mm-thick electrodeelectrolyte assemblies in electrochemical cells. In situ ASAXS experiments in electrochemical cells can thus be performed under electrochemical working conditions. Ex situ studies of electrodes, removed from the potential environment in the electrochemical cell, are of limited value, since changes can occur during the washing and drying procedures.

In situ ASAXS (Haubold et al., 1999) was successfully applied to study electrochemical oxidation of methanol as in Direct Methanol Fuel Cells and to trace the formation and oxidation of intermediate products on the surface of carbon supported Pt catalyst particles, as a function of the applied electrochemical potential. Catalysis on the reduced metallic platinum particles was found to become hindered by the formation of a shell of oxidised platinum on their surface. ASAXS was used here as an in situ monitor. Fig. 2 illustrates in situ results of a cyclic potential sweep.

The integrated SAXS drops sharply at about $600 \mathrm{mV}$ in the anodic sweep and remains at a low value up to $400 \mathrm{mV}$ in the reverse sweep in the cathodic direction. The ASAXS analyses at $250 \mathrm{mV}$ and $1100 \mathrm{mV}$ show changes in the particle size distribution. Only at the lower potential is the Pt catalyst found in reduced metallic particles with radius of about $9 \AA$. At the higher potential, the particle size distribution is shifted to larger radii of about $11 \AA$ : $\mathrm{Pt}$ atoms on the particle surface moved outward to form an oxide shell with a decreased number density of Pt atoms. This is confirmed by in situ measurement of the "white line" absorption in the XANES Pt-L3 absorption spectrum, which gives information on the Pt dband occupancy.

\subsection{Composition of phases or determination of specific volumes in two-phase materials}

If weak diffuse scattering is excluded, the small-angle intensity is the product of the contrast between the two phases $\alpha$ and $\beta$, and of the geometrical structure factor $S_{\phi}(q)$, i.e., the Fourier transform of pair correlations inside a phase (Patterson function):

$$
\mathrm{d} \sigma / \mathrm{d} \Omega(q)=\left(\rho_{\alpha}(E)-\rho_{\beta}(E)\right)^{2} S_{\phi}(q)
$$

where $\rho$ is the electronic density of a phase, the sum of the contributions of all elements. The applications can be classified into two families, an example of each being given below:

(i) Atomic volumes are known, as in the case of phase separation on a lattice: atomic concentrations are equal to volumetric ones and their sum is equal to 1 .

(ii) If not, information on specific volumes can be obtained if concentrations are known or vice versa, thereby requiring an extra experiment.

The determination of the composition of metastable precipitates in an age hardenable $\mathrm{Al} \mathrm{Zn} \mathrm{Cu} \mathrm{Mg}$ alloy illustrates the first case. Optimum mechanical properties are obtained not by formation of GP zones at room temperature but after a heat treatment that triggers germination and growth of an intermediate phase. In ternary $\mathrm{Al} \mathrm{Mg}$ $\mathrm{Zn}$ alloys, the intermediate phase is $\mathrm{MgZn}_{2}$. For the quaternary alloy, EXAFS measurements fail to decide whether or not precipitates contain $\mathrm{Cu}$.

It was therefore decided to perform ASAXS measurements. With Al as a reference, the two unknown quantities are the ratios

$\left(c_{\mathrm{p}}-c_{\mathrm{m}}\right)_{\mathrm{Zn}} /\left(c_{\mathrm{p}}-c_{\mathrm{m}}\right)_{\mathrm{Mg}}$ and $\left(c_{\mathrm{p}}-c_{\mathrm{m}}\right)_{\mathrm{Cu}} /\left(c_{\mathrm{p}}-c_{\mathrm{m}}\right)_{\mathrm{Mg}}$, where $c_{\mathrm{p}}$ and $c_{\mathrm{m}}$ are respectively the concentration in the precipitates and in the matrix for the given element. They may in principle be determined by performing ASAXS experiments near both $\mathrm{Cu}$ and $\mathrm{Zn} \mathrm{K}$ edges. But since the atomic numbers of $\mathrm{Al}$ and $\mathrm{Mg}$ are very close, contrast differentiation is expected to be difficult: ASAXS spectra were therefore recorded at numerous different energies and integrated intensities in a given $q$ range were calculated in order to improve the precision. Fig. 3 compares the best fit (dashed line) to experimental values (circles). As second hypothesis, it was assumed that precipitates had a composition $\mathrm{Mg}\left(\mathrm{Zn}_{2-x} \mathrm{Cu}_{x}\right)$, with $0<x<2$, which reduces to the number of unknown parameters to one. The fit 

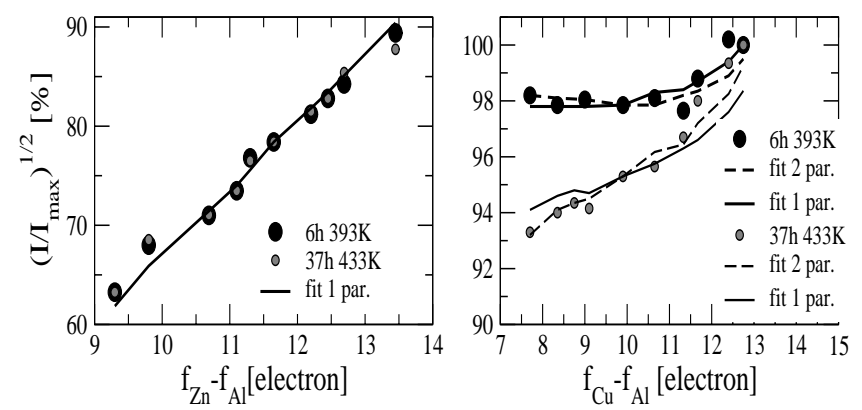

Figure 3

ASAXS intensities as function of the $\mathrm{Cu}$ and $\mathrm{Zn}$ contrast with respect to $\mathrm{Al}$ for the two different ageing treatments indicated (precipitate sizes $1.2 \mathrm{~nm}$ and $4.5 \mathrm{~nm}$, respectively). Near the $\mathrm{Cu}$ edge, the two parameter fit is slightly different from that with a single parameter and also depends on ageing; near the $\mathrm{Zn}$ edge, however, the fits are undistinguishable and, moreover, are independent of ageing.

(continuous line) is as good as the previous one. The final result is as follows: the amount of $\mathrm{Cu}$ in precipitates is small, ranging from negligible in the early stages up of $7-8 \%$ at later stages. In this experiment, the precision of the ASAXS measurements $(\sim 0.5 \%)$ is not limited by statistics but by the stability of the monochromator and of the detection.

For structural characterization of amorphous materials, ASAXS is a powerful experimental technique, because other techniques like EXAFS or WAXS provide no information about structure on length scales larger than $1 \mathrm{~nm}$. Fig. 4 displays results obtained from an ASAXS measurement of an amorphous silicon-germanium alloy, performed in the vicinity of the $\mathrm{Ge}-\mathrm{K}$ edge at $11103 \mathrm{eV}$. The scattering curve with the higher cross section is the total scattering obtained from the alloy at $10008 \mathrm{eV}$. The lower scattering curve, corresponding to the separated scattering due to Ge, was obtained by subtracting the two scattering curves measured at 10008 and $11098 \mathrm{eV}$ : this contribution from non-uniformly distributed Ge in the amorphous matrix is substantial. The solid line is the fitted square-Lorentzian function of a two-phase mixture with correlation length $\xi$ : the structure is considered as consisting of a homogeneous material in which a second phase with a variable and undetermined shape is distributed. Equations (2) and (3) summarize the relevant physical parameters, that can be obtained from the lower scattering curve of Fig. 4.

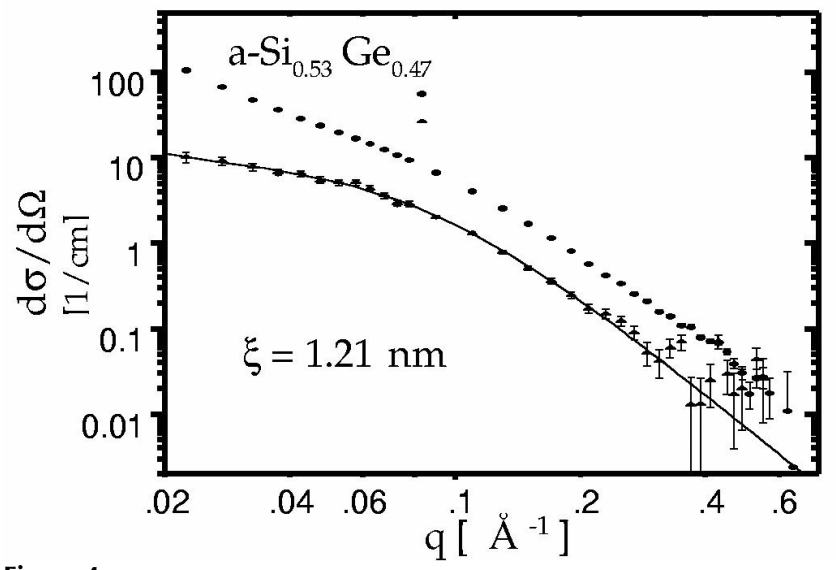

Figure 4

Total (upper curve) and separated scattering curve of an amorphous silicongermanium alloy.
The scattered intensity given in equation (2) contains the structural information i.e. the correlation length $\xi$ of a two-phase mixture, $\Phi$ the volume fraction of the phase $\alpha$, while $r_{0}$ is the scattering length of the electron.

$$
\begin{gathered}
\mathrm{d} \sigma / \mathrm{d} \Omega(q, E)=8 \pi r_{\mathrm{o}}^{2} \Phi(1-\Phi)\left(\rho_{\alpha}(E)-\rho_{\beta}(E)\right)^{2} \xi^{2} /\left(1+\xi^{2} q^{2}\right)^{2} \\
\text { with } \rho_{\alpha}(E)-\rho_{\beta}(E)= \\
\left(n_{\alpha} X_{\alpha}^{\mathrm{Si}}-n_{\beta} X_{\beta}^{\mathrm{Si}}\right) f_{\mathrm{Si}}(E)+\left(n_{\alpha} X_{\alpha}^{\mathrm{Ge}}-n_{\beta} X_{\beta}^{\mathrm{Ge}}\right) f_{\mathrm{Ge}}(E)
\end{gathered}
$$

Equation (3) contains important quantitative parameters (like the average atomic densities $n_{\alpha}, n_{\beta}$ and the chemical concentrations $X_{i}{ }^{j}$ of the two phases) that can be determined by extended ASAXS measurements in conjunction with density measurements. Fig. 5 illustrates the improvement in precision when, by virtue of the continuously tuneable wavelength, is obtained by over-determining the number of equations with respect to the number of unknown parameters.

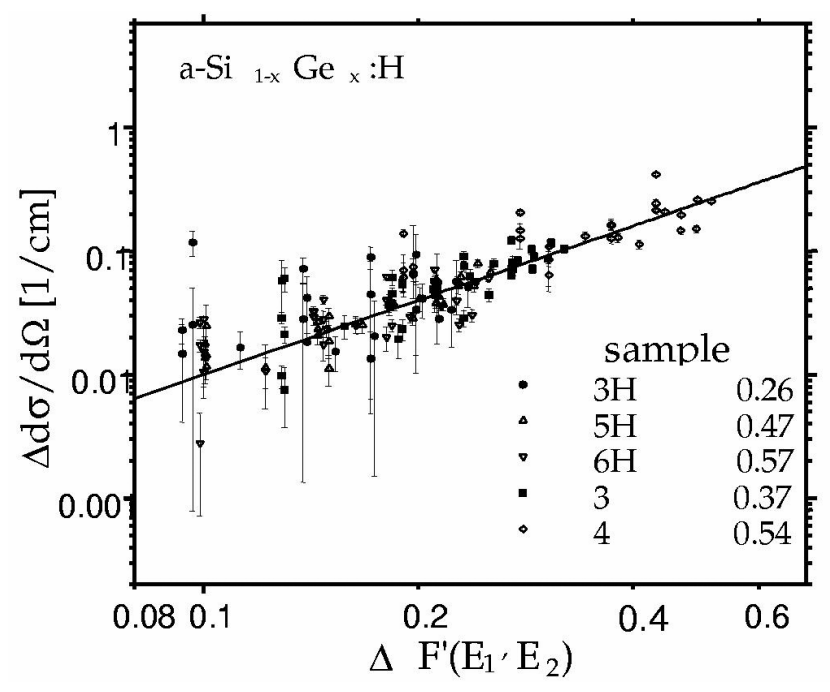

Figure 5

Energy dependence of the separated scattering (at $q=0$ ) obtained from five alloys with Ge-concentrations $x$. The samples with the signature $\mathrm{H}$ are the alloys prepared under high hydrogen dilution.

Table 1 lists the ASAXS results obtained from three alloys prepared under high hydrogen dilution. For Ge concentrations larger than $20 \%$, inhomogeneities start to develop with correlation lengths slightly greater than the atomic size. The $\alpha$-phase extends over about $90 \%$ of the volume with a Ge concentration somewhat bigger than the overall alloy. This can be interpreted as dense regions containing enhanced numbers of homopolar Ge bonds with average sizes between 10 and $12 \mathrm{~nm}$, surrounded by a small volume fraction of low density, H-rich regions containing much less Ge (sometimes none) with average sizes from 0.6 to $1.6 \mathrm{~nm}$ (Goerigk and Williamson, 2001). 
Table 1

Quantitative properties obtained from ASAXS measurements on three alloys. Germanium and silicon concentrations (Equation 3) are related by $X_{\alpha, \beta}{ }^{\mathrm{Si}}=1-X_{\alpha, \beta}{ }^{\mathrm{Ge}}$. The densities $\eta_{\alpha}=n_{\alpha} / n, \eta_{\beta}=n_{\beta} / n$ are the relative average atomic number densities of the $\alpha$ and $\beta$ phases divided by the average atomic density $n$ of the entire alloy. The estimated errors are about 0.01 .

\begin{tabular}{ccccccccc}
\hline$X$ & $X_{\alpha}{ }^{\mathrm{Ge}}$ & $X_{\beta}{ }^{\mathrm{Ge}}$ & $\eta_{\alpha}$ & $\eta_{\beta}$ & $\begin{array}{c}\xi \\
{[\mathrm{nm}]}\end{array}$ & $\begin{array}{c}\xi_{\mathrm{a}} \\
{[\mathrm{nm}]}\end{array}$ & $\begin{array}{c}\xi_{\beta} \\
{[\mathrm{nm}]}\end{array}$ & $\Phi$ \\
\hline 0.26 & 0.27 & 0. & 1.036 & 0.34 & 0.55 & 11.0 & 0.58 & 0.95 \\
0.47 & 0.50 & 0.08 & 1.055 & 0.29 & 1.25 & 17.9 & 1.34 & 0.93 \\
0.57 & 0.60 & 0.40 & 1.070 & 0.58 & 1.36 & 9.7 & 1.58 & 0.86 \\
\hline
\end{tabular}

\subsection{Coupling ASAXS/AWAXS or ASAXS/AXRD}

A new method of extracting displacements from chemical variation of precipitates combines diffuse scattering near the origin of reciprocal space and near Bragg peaks. This separation uses the properties of symmetry and periodicity of the successive terms of the expansion of the scattering equation (Lyon et al., 2000). In a Cu$\mathrm{Ti}$ alloy, the regularity of the pattern (tweed pattern) was explained by a short range repulsive elastic interaction, and an attraction at larger distances. In this conference, Guillon et al. (2001) present the more complex case of the ternary $\mathrm{Cu}-\mathrm{Ni}-\mathrm{Fe}$ alloy, which is a prototype of continuous phase separation systems.

The example described hereafter concerns the early stages of phase separation/ordering in a model $\mathrm{Ni}-\mathrm{Cr}$-Al superalloy by ASAXS and AXR Diffraction (M. Delheusy, F. Bley, J.P. Gaspard, F. Livet, J.P. Simon; recent experiments). The question to be answered was: Which type of fluctuations (order fluctuations or composition fluctuations) occurs first?

By probing numerous energies with ASAXS near both the $\mathrm{Cr}$ and the Ni edges (Fig. 6a), the formalism of "pair correlation functions" and "partial structure factors" (PSF) gives the most complete structural information available from (non-coherent) elastic scattering. The main difficulty arises from the resolution of an illconditioned system of linear equations (small contrast variation), but with the possibility of sampling over-determination. The analysis uses the cross-checking method for testing the compatibility between different intensities proposed by O. Lyon (1993).

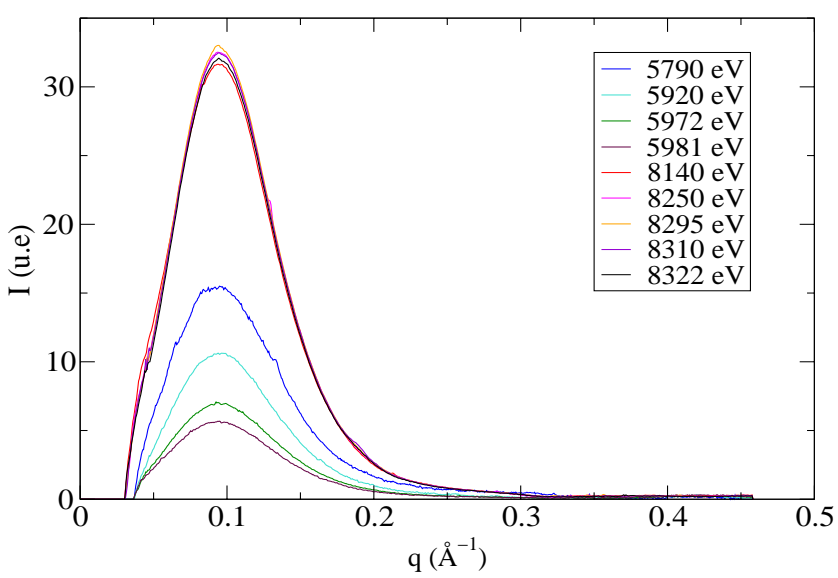

Figure 6

SAXS profile in a Ni-Cr-Al quenched from the homogeneous state and aged $8 \mathrm{~h}$ at $873 \mathrm{~K}$ for the energies indicated; note that the shape is identical and that the profiles near the Ni edge $(8333 \mathrm{eV})$ are almost identical.
The resulting PSFs (Fig. 7) are compatible with a two phase pattern and, independently of the aging between $0.33 \mathrm{~h}$ and $8 \mathrm{~h}$ at $873 \mathrm{~K}$ (with segregation increasing by a factor 2), there is almost no segregation of $\mathrm{Ni}$ : $\mathrm{Al}$ replaces $\mathrm{Cr}$ in the precipitates. This reaction occurs with a weak increase of the volume fraction and a progressive enrichment in $\mathrm{Al}$ : it differs from classical nucleation, growth and coarsening processes.

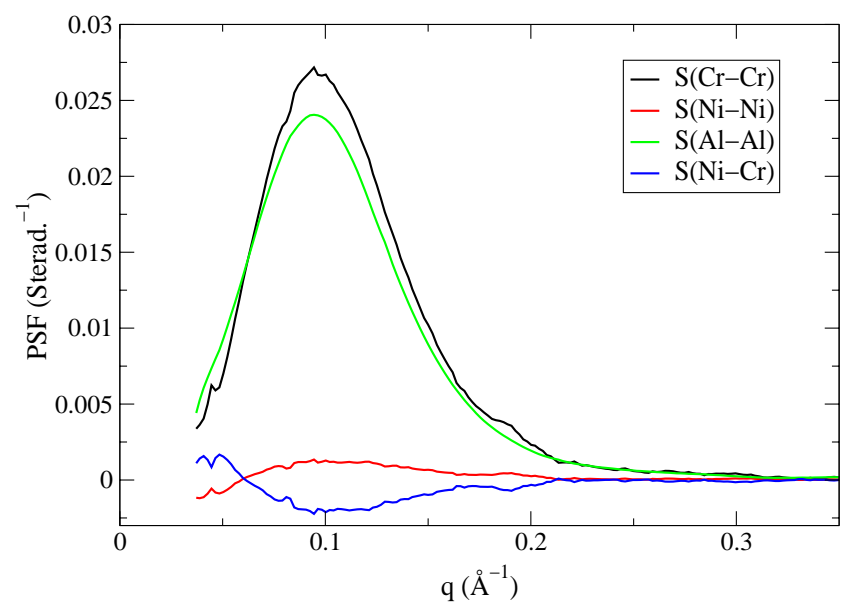

Figure 7

Corresponding PSF; as expected, they are positive except, at small angles, for $S_{\mathrm{NiNi}}$, which is elsewhere negligible (this comes from imperfect correction of the very small angle signal from pores, surfaces or large defects).

The 100 superstructure peak (Fig. 8) has been recorded by powder diffraction at different energies. From their width, the size is deduced using the Scherrer relation: the size of these ordered domains is almost the same as that measured by SAXS: a correspondence exists between precipitates and ordered domains. The partial long range order parameters can be deduced from the dependence of intensity on photon wavelength (Marty et al., 1990): precipitate ordering is complete from the earliest stage studied; the decrease of the $\mathrm{Cr}$ order parameter and increase in that of $\mathrm{Al}$ means that $\mathrm{Ni}_{3}(\mathrm{Al}-\mathrm{Cr})$ precipitates with $\mathrm{Ll}_{2}$ structure absorb $\mathrm{Al}$ from the matrix and reject $\mathrm{Cr}$.

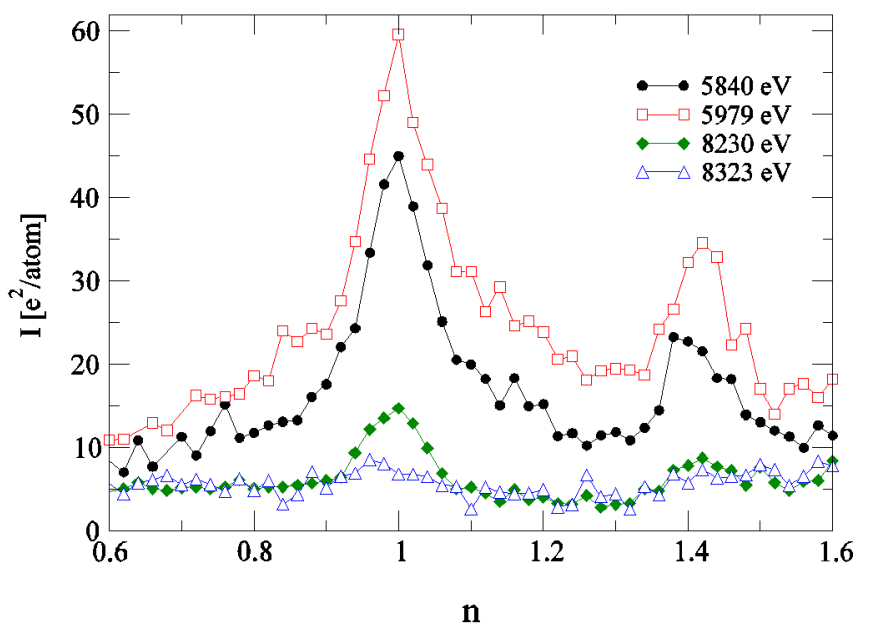

Figure 8

Superstructure profiles for 100 (at $n=1$ ) and 110 (at $n=\sqrt{ } 2$ ) Bragg peaks for the same samples. 


\section{Conclusion}

With synchrotron radiation, ASAXS has become a mature, quantitative technique, used in numerous investigations in materials science and catalyst research. Synchrotron radiation is essential in detecting weak small angle scattering signals (e.g., from diluted or amorphous alloys) and its continuous X-ray spectrum enables anomalous signals to be used for the characterisation of the different chemical constituents.

ASAXS is an alternative technique for retrieving information that is inaccessible by standard small angle scattering methods. Possible applications are:

(i) Contrast enhancement, combined with the separation of different kinds of inhomogeneities, e.g. for following decomposition kinetics in $\mathrm{Cu}$ Co alloys. The ASAXS technique is tailored for separating the SAXS signal of tiny metal particles from the much larger background signal of its substrate and thus to follow in-situ their catalytic effect in an experimental device resembling commercial fuel cells.

(ii) Compositions of phases in two-phase materials, e.g., determining nanoprecipitate composition such as GP zones in alloys. In the case of amorphous materials, ASAXS measurements have been combined with density measurements to determine the volume fractions, densities and chemical concentrations of the two phases.

(iii) If combined with other scattering/diffraction techniques, it has been used for disentangling ordering from precipitation in superalloys.

(iv) It may be also associated with grazing incidence (Kutsch et al., 1997) or with X-ray magnetic dichroism (Fischer et al., 1998).

Recently, it has been employed in soft condensed matter, e.g., to localize the distribution of counterions (from $\mathrm{Cs}, \mathrm{Rb}, \mathrm{I}, \mathrm{Ba} . .$. ) around polyelectrolytes (Guilleaume et al., 2002 or Dingenouts et al., this conference).

\section{References}

Benedetti, A., Polizzi, S., Riello, P., Pinna, F. \& Goerigk, G. (1997). J. Catalysis, 171, 345-348.

Benedetti, A., Bertoldo, L., Canton, P., Pinna, F., Goerigk, G., Riello, P. \& Polizzi, S. (1999). Catalysis Today, 49/4, 485-489.

Bönnemann, H., Waldöfner, N., Haubold, H.-G. \& Vad, T. (2002). Chem. Mater., 14, 1115-1120.

Bota, A., Goerigk, G., Drucker, T., Haubold, H.-G. \& Petro, J. (2002). J. Catalysis, 205, 354-357.

Cromer, D. T. \& Liberman, D. (1981). Acta Cryst. A 37, 267-268.

Cross, J.O., Newville, M., Rehr, J.J., Sorensen, L.B., Bouldin, L.B., Watson, G., Gouder, G., Lander, G.H. \& Bell, M.I. (1998). Phys. Rev. B 58, 1121511225 .

Ferrer, J.-L., Simon, J.-P., Bérar, J.-F., Caillot, B., Fanchon, E., Kaikati, O., Arnaud, S., Guidotti, M., Pirocchi, M. \& Roth M. (1998). J. Synchr. Rad. 5, 1346-1356.

Fischer, P., Zeller, R., Schutz, G., Goerigk, G., Haubold, H.G., Pruegl, K. \& Bayreuther, G. (2000). J. Appl. Phys. 83, 7088-7090.

Goerigk, G., Haubold, H.-G. \& Schilling, W. (1997). J. Appl. Cryst. 30, 1041-1047.

Goerigk, G. \& Williamson, D.L.(2001). J. Non-Cryst. Solids 281, 181-188.

Guilleaume, B., Blaul, J, Ballauff, M., Wittemann, M., Rehahn, M. \& Goerigk, G. (2002). Eur. Phys. J. E8, 299-309.

Haubold, H.-G., Gruenhagen, K., Wagener, M., Jungbluth, H., Heer, H., Pfeil, A., Rongen, H., Brandenberg, G., Moeller, R., Matzerath, J., Hiller, P. \& Halling, H. (1989). Rev. Sci. Instr. 60, 1943-1946.

Haubold, H.-G., Gebhardt, R., Buth, G. \& Goerigk, G. (1994). in "Resonant Anomalous X-Ray Scattering", edited by Materlik G., Sparks C. J. \& Fischer K.; Elsevier Science, 295-304.

Haubold H.-G., Hiller P., Jungbluth H. \& Vad T. (1999). Jpn. J. Appl. Phys.
Suppl. 38-1, 36-39.

Haubold H.-G., Wang X. H. (1995). Nucl. Instr. and Meth. in Phys. Res. B 97, $50-54$

Haubold H.-G., Wang X. H., Jungbluth H., Goerigk G., Schilling W. (1996). J. of Molecular Structure 383, 283-289.

Kutsch B., Lyon O., Schmitt M., Menning M. \& Schmidt H. (1997). J. Appl. Cryst. 30, 948-956.

Lyon O. (1993). in "Methods in the Determination of Partial Structure Factors" edited by Suck J.B., Chieux P., Raoux D. \& Riekel C.; World Scientific, 142-150.

Lyon O., Guillon I. \& Servant C. (2001). J. Appl. Cryst. 34, 484-492.

Marty A., Bessiere M., Bley F., Calvayrac Y. \& Lefebvre S. (1990). Acta Metall. Mater. 38, 345-350.

Polizzi S., Riello P., Balerna A. \& Benedetti A. (2001). Phys. Chem. Chem. Phys. 3, 4614-4619.

Rasmussen F. B., Molenbrock A. M., Clausen B. S. \& Feidenhans'1 R. (2000). J. Catalysis, 190, 205-208.

Simon J.-P. \& Lyon O. (1994). in "Resonant Anomalous X-Ray Scattering", edited by Materlik G., Sparks C. J. \& Fischer K.; Elsevier Science, 305-322.

Simon J.-P., Arnaud S., Bley F., Bérar J.F., Caillot B., Comparat V., Geissler E., de Geyer A., Jeantet P., Livet F. \& Okuda H. (1997). J. Appl. Cryst. 30, 900-904.

Stuhrmann H.-B. (1985). Advances in Polymer Sciences 67, 123-163.

Vad T., Haubold H.-G., Waldöfner N. \& Bönnemann H. (2002). J. Appl. Cryst., 35, 459-470. 